\title{
CRISE : QUELQUES RÉFLEXIONS SYSTÉMIQUES SUR L'ANTHROPOCÈNE
}

\author{
Author(s) / Auteur(s) : \\ Honorary President of the Argentine Group for the Study of Systems and Cybernetics (GESI), \\ the Latin American Society of Systemics (ALAS), \\ Honorary Professor the Instituto Tecnológico de Buenos Aires, Argentina (ITBA) \\ francoischg@fibertel.com.ar
}

\section{Résumé :}

Avant la globalisation les crises résultaient d'abus écologiques locaux ou régionaux, de conflits de leadership, de catastrophes climatiques locales ou régionales, de grandes épidémies ou pandémies. L'humanité du début du 21 ème siècle est confrontée pour la première fois à une situation de changement global à l'échelle planétaire. L'origine de ce changement est endogène à l'espèce humaine. L'évolution scientifique et technique a doté l'humanité de puissants moyens d'action qui l'ont transformée en un acteur de poids dans l'écologie planétaire. Cette transformation est une conséquence de facteurs fondamentaux qui se sont renforcés réciproquement. L'utilisation des énergies fossiles retourne à l'environnement des quantités gigantesques d'énergie solaire fossilisée sous forme végétale durant les milliards d'années des ères géologiques primaire, secondaire et tertiaire. La conséquence inévitable est et sera de plus en plus, le réchauffement du climat planétaire. Ce changement profond pourrait sûrement causer de profonds troubles adaptatifs dans toutes les sociétés humaines. C'est en ce sens que le mot «crise» acquiert son sens plein et sinistre. Une crise financière n'est rien d'autre que la répétition d'un épisode psychosociologique, récurrent dans l'évolution de l'économie. Une conséquence peu remarquée est que l'homme (biologique, psychosocial) est devenu une "pièce» d'une immense machine, à l'échelle planétaire, ses activités étant de plus en plus conditionnées par cette machine créée par luimême. Le concept d'avantage concurrentiel domine généralement la pensée économique. Cette façon de voir reflète la situation historique de l'humanité. Jusqu'à nos jours, l'espèce humaine pouvait progresser sans limites vers la plus grande utilisation de son environnement planétaire. Les déchets n'avaient pas une signification écologique négative. Le but le plus sensé et le plus approprié n'est certainement pas de conduire l'humanité entière à partager les déchets qui caractérisent pour l'instant les sociétés considérées comme développées. Un objectif rationnel serait de garantir à l'humanité tout entière un niveau de vie le plus satisfaisant possible, basé sur des ressources planétaires réellement renouvelables assumant la nécessité de garder la planète habitable.

\section{Mots-clés :}

croissance, crise sociétale, développement durable, écologie, globalisation, humanité, idéologie, rétro-action

\begin{abstract}
Resumen:
Antes de la globalización, las crisis se debían a abusos medioambientales locales o regionales, conflictos de liderazgo, desastres climáticos locales o regionales, grandes epidemias o pandemias. La humanidad de comienzos del siglo 21 se enfrenta por primera vez con una situación de cambio global a escala planetaria. El origen de este cambio es endógeno a la especie humana. La evolución científica y tecnológica ha dotado a la humanidad de poderosos medios de acción que la han transformado en un actor importante en la ecología planetaria. Esta transformación es una consecuencia de factores fundamentales que se han reforzado mutuamente. El uso de energías fósiles devuelve al medio ambiente cantidades gigantescas de energía solar fosilizada en forma vegetal durante los miles de millones de años de las eras geológicas primarias, secundarias y terciarias. La consecuencia inevitable es y será cada vez más, el calentamiento global del clima. Este cambio profundo podría seguramente causa serios desórdenes de adaptación en todas las sociedades humanas. Es en este sentido que la palabra "crisis" adquiere su significado pleno y siniestro. Una crisis financiera no es más que la repetición de un episodio psicosociológico también recurrente en la evolución de la economía. Una consecuencia notable es que el hombre (biológico, psicosocial) se ha convertido en una "pieza" de una máquina enorme, a escala planetaria, y sus actividades están cada vez más condicionadas por esta máquina creada por él mismo. El concepto de ventaja competitiva generalmente domina el pensamiento económico. Esta forma de pensar refleja la situación histórica de la humanidad. Hasta hoy, la especie humana podría progresar sin límites hacia un mayor uso de su entorno planetario. Los residuos no tuvieron un significado ecológico negativo. El
\end{abstract}


objetivo más sensato y apropiado, ciertamente, no es llevar a toda la humanidad a compartir los desechos que por hiper consumo caracterizan por el momento a las sociedades consideradas como desarrolladas. Un objetivo racional sería garantizar a toda la humanidad un nivel de vida lo más satisfactorio posible, basado en recursos planetarios verdaderamente renovables, asumiendo la necesidad de mantener al planeta habitable.

\title{
Palabras clave:
}

crecimiento, crisis social, desarrollo sostenible, ecología, globalización, humanidad, ideología, retroacción

\begin{abstract}
Summary:
Prior to globalization, crises were due to local or regional environmental abuses, leadership conflicts, local or regional climatic disasters, major epidemics or pandemics. The humanity of the beginning of the 21 st century is facing for the first time a situation of global change on a planetary scale. The origin of this change is endogenous to the human species. Scientific and technological evolution has endowed humanity with powerful means of action that have transformed it into an important actor in the planetary ecology. This transformation is a consequence of fundamental factors that have reinforced each other. The use of fossil fuels returns to the environment gigantic amounts of solar energy fossilized in vegetable form during the billions of years of the primary, secondary and tertiary geological ages. The inevitable consequence is and will be more and more, global climate warming. This profound change could it surely causes serious adjustment disorders in all human societies. It is in this sense that the word "crisis" acquires its full and sinister meaning. A financial crisis is nothing more than the repetition of a psychosociological episode also recurrent in the evolution of the economy. A remarkable consequence is that man (biological, psychosocial) has become a "piece" of a huge machine, on a planetary scale, and his activities are increasingly conditioned by this machine created by himself. The concept of competitive advantage generally dominates economic thinking. This way of thinking reflects the historical situation of humanity. Until today, the human species could progress without limits towards greater use of its planetary environment. The waste did not have a negative ecological significance. The most sensible and appropriate objective, certainly, is not to lead all of humanity to share the waste that, by hyper-consumption, characterizes, for the moment, the societies considered as developed. A rational objective would be to guarantee to all humanity a level of life as satisfactory as possible, based on truly renewable planetary resources, assuming the need to keep the planet habitable.
\end{abstract}

\section{Keywords:}

growth, social crisis, sustainable development, ecology, globalization, humanity, ideology, feedback

\section{QU'EST-CE QU'ON APPELLE "CRISE" ?}

Le mot lui-même est très dévalué et a perdu beaucoup de sens à cause de son utilisation constante en relation avec une situation compliquée. En même temps, il est indubitable que nous sommes au milieu d'une profonde transformation globale dans pratiquement tous les domaines de la vie des sociétés. Ce changement a commencé en Europe occidentale il y a plusieurs siècles et s'est accéléré de plus en plus. Les sociétés du passé étaient plus stables que les sociétés actuelles parce que le mode de vie fondamental variait peu d'un siècle à l'autre. On pourrait parler, par exemple, d'une ancienne culture ou civilisation du blé et de la vigne, d'une civilisation du riz, d'autres du maïs et (en Afrique) des cultures de manioc. Il y avait aussi des cultures d'oasis et de chameaux, des cultures de pêche et même des cultures prédatrices (les peuples barbares vivent des raids au détriment des cultures les plus avancées). Dans ces cultures, les crises résultaient d'abus écologiques locaux ou régionaux (qui affectaient la subsistance de base), de conflits de leadership, de catastrophes climatiques locales ou régionales (sécheresses, périodes glaciaires) de grandes épidémies ou pandémies. 


\section{L'HOMME, FACTEUR GLOBAL DE CRISE}

L'humanité du début du $21^{\mathrm{e}}$ siècle est confrontée pour la première fois à une situation de changement global à l'échelle planétaire. L'origine de ce changement est endogène à l'espèce humaine: depuis le XVIIe siècle, l'homme a été de plus en plus introduit dans l'écologie planétaire comme un grand facteur de changement, de plus en plus puissant. L'évolution scientifique et technique a doté l'humanité de puissants moyens d'action qui l'ont transformée en un acteur de poids dans l'écologie planétaire globale.

Cette transformation est une conséquence de facteurs fondamentaux qui se sont renforcés réciproquement :

- Une vision rationnelle et scientifique du monde apparu et développé en Europe, depuis le Moyen Âge et la Renaissance

- Le développement, lent au début puis en accélération constante, de techniques de plus en plus complexes, qui conduisent toutes à une «artificialisation» ou à une «artifactisation» pour utiliser un néologisme lourd mais utile

- La mobilisation croissante (grâce au progrès technologique) de sources d'énergie jusqu'alors inconnues.

Ce processus a commencé en Europe de l'Ouest avec l'exploitation de plus en plus intensive des gisements de charbon, jusqu'à quasi- épuisement. Il a continué au $20^{\mathrm{e}}$ siècle d'une manière similaire avec l'utilisation d'autres combustibles fossiles: le pétrole et le gaz naturel (également épuisable, sans aucun doute). Sans s'en rendre compte, l'homme est ainsi devenu un facteur d'évolution écologique accélérée, à l'échelle mondiale. L'extraction du charbon et l'exploitation pétrolière et gazière continuent de retourner à l'environnement planétaire, sans cesse, des quantités gigantesques d'énergie solaire fossilisée sous forme végétale durant les milliards d'années des ères géologiques: primaire, secondaire et tertiaire. La conséquence inévitable est et sera de plus en plus dans le futur, le réchauffement du climat planétaire. Ce changement profond pourrait sûrement aussi causer de profonds troubles adaptatifs dans toutes les sociétés humaines.

C'est en ce sens que le mot «crise» acquiert son sens plein et sinistre.

Face à cette perspective, toute l'humanité devrait réagir de différentes manières :

- Étudier en profondeur les effets globaux de la consommation massive de combustibles fossiles

- Prévoir des délais pour les épuiser le plus précisément possible

- Rechercher des sources alternatives d'énergies renouvelables

- Planifier la transition des combustibles fossiles aux énergies renouvelables pour assurer la stabilité économique, démographique et socioculturelle à long terme.

\section{La crise financière: un phénomène psychosociologique typique.}

C'est plus qu'un problème économique, mais cela se reflète mal, en particulier dans le vocabulaire qui utilise le mot "investisseurs" pour qualifier la majorité des "spéculateurs" qui achètent et vendent des "titres" sur le marché boursier, dans l'espoir de faire une "différence", ou de "tirer profit". Évidemment, les opinions divergentes sur le présent et le futur. La valeur d'une action sur le marché boursier implique parfois des critères subjectifs et moraux, plutôt que des termes rationnels. C'est ce que reflète le mot "spéculateur» (L'histoire des grandes et soudaines perturbations des différents marchés au cours des derniers siècles reflète très clairement ce phénomène psychosociologique récurrent dans toutes les cultures modérément avancées). La crise financière actuelle n'est rien d'autre que la répétition d'un épisode psychosociologique aussi récurrent dans l'évolution de l'économie. C'est un phénomène évolutionnaire peu perçu et compris, étant donné que l'homme a vécu jusqu'à un passé récent dans le «présent», c'est-à-dire sans se rendre compte des profondes transformations à long terme que ses initiatives introduisent dans leurs relations complexes avec son environnement. Même aujourd'hui, la grande majorité des gens vit existentiellement dans le présent avec peu de mémoire du passé et peu de projections dans le futur. Seuls des objectifs à court terme limités et partiels sont intéressants, sans vision et sans souci des conséquences globales ultérieures possibles ou probables. 
L'opinion actuelle la plus répandue est que le «développement» (économique et social) doit se développer et se poursuivre sans limites pour atteindre l'ensemble de l'humanité. Est étiqueté comme "pessimiste" ou "alarmiste" qui met en garde contre les limitations probables du développement par rapport aux ressources planétaires. Un article récent a déclaré que: "... nous ne progressons pas dans la croissance et le développement de la société humaine au rythme requis". A cet égard, s'il est souhaitable d'extraire de leur misère des populations et des fractions de populations - même dans le monde développé -, le but le plus sensé et le plus approprié n'est certainement pas de conduire l'humanité entière à partager déchets qui caractérisent pour l'instant les sociétés considérées comme développées. Si l'on prend en compte la dimension à moyen et long terme du futur, un objectif rationnel serait de garantir à l'humanité tout entière un niveau de vie le plus satisfaisant possible, basé sur des ressources planétaires réellement renouvelables assumant la nécessité de garder la planète habitable. Les ressources non renouvelables devraient être utilisées pour «amorcer» un système de production autosuffisant, basé sur des ressources renouvelables. De bons exemples seraient l'utilisation de carburants non renouvelables comme contribution à la construction de centrales hydroélectriques, ou leur utilisation pour l'amélioration et la généralisation des systèmes de captage de l'énergie solaire. La dynamique de l'utilisation des combustibles fossiles L'utilisation croissante et massive des combustibles fossiles du Moyen Âge, d'abord en Europe occidentale, a été le principal facteur déclenchant (l'autre innovation technique) de l'énorme développement démographique, économique et social au cours de la siècles récents. L'utilisation des énergies fossiles était d'abord un phénomène auto-entretenu et ensuite auto-accélérateur.

Cela n'a été possible que grâce à l'existence d'un gigantesque «trésor» énergétique accumulé sous forme fossile pendant plusieurs centaines de millions d'années (de l'ère primaire à la période géologique la plus récente). La production de réserves d'énergie fossile étant un processus extrêmement lent, on peut considérer que le «trésor» énergétique n'est pas reconstitué de manière appréciable alors que l'humanité le consomme de plus en plus rapidement. La conclusion évidente est que le "trésor", au rythme actuel de son utilisation, sera inévitablement épuisé dans une période probablement de quelques décennies, selon le "Hubber Peak", caractérisé par une courbe de croissance à long terme exponentielle jusqu'à un maximum très élevé, suivi d'une chute presque totale sur une période extrêmement courte. Finalement, nous arrivons au critère que le «trésor» fossile devrait être appliqué, au moins en partie, pour créer l'infrastructure et les méthodes pratiques d'utilisation des énergies renouvelables, applicables à toutes les utilisations variées que nous faisons des combustibles fossiles, "amorçage", comme nous l'avons dit plus haut, la pompe à énergie renouvelable.

De même, une description précise et un équilibre des effets collatéraux (écologiques, sociopolitiques, etc.) de l'utilisation massive des combustibles fossiles devraient être établis et, à terme, la réparation des dommages collatéraux considérables produits devrait être abordée.

\section{L'instabilité politique mondiale potentielle par rapport à l'existence et à la consommation de combustibles fossiles.}

Bien que généralement méconnu, le pouvoir politique des nations dépend plus ou moins étroitement de l'exploitation du potentiel énergétique qu'elles sont capables de contrôler et d'utiliser. Aucune nation moderne ne peut être puissante, encore moins se maintenir en tant que telle si elle n'a pas les énergies fossiles qui assurent ses bases matérielles productives et le maintien de ses infrastructures économiques et sociopolitiques. Cette question a une dimension temporelle très importante . Le pouvoir politique international d'un pays n'est garanti et solide que dans la mesure où il a une autonomie énergétique; c'est-à-dire, encore principalement le contrôle des réserves sur leur propre territoire ... ou plus difficile à garantir et à maintenir, sur le territoire des autres états. Considérant que les combustibles fossiles localisés ou à découvrir seront épuisés lorsqu'ils seront exploités, tout pays devrait prévoir son autonomie: Prévoir l'épuisement des réserves d'énergie non renouvelables dont elle dépend (notamment les réserves propres non territoriales). Prévoir le remplacement qualitatif et quantitatif de ces énergies par d'autres énergies renouvelables et techniquement utilisables (par exemple remplacer à l'avenir massivement le moteur diesel par d'autres types de moteurs qui n'utiliseraient pas de combustibles fossiles?) 
Une prospective (ou prévision) adéquate peut garantir une transition vers d'autres formes d'organisation économique et productive, et éviter d'éventuelles violences socio- économiques. convulsions politiques mises en scène par des masses amenées à des conditions de misère et de pauvreté.

\section{LA COMPÉTITIVITÉ DES NATIONS : PRÉSENT ET FUTUR}

Tandis que la science et ses applications technologiques ont été à la base de la compétitivité des nations Si la science et ses applications techniques ont été à la base de la compétitivité des nations dans l'économie mondiale, il est clair que rien n'a été, n'est et ne sera possible sans garantie d'approvisionnement permanent des matières premières, dont l'exploration nécessite toujours de l'énergie.

\section{Matières premières}

La question de la compétitivité se résume finalement dans la fourniture gratuite et suffisante de matières premières, et plus encore, d'énergie. Étant donné que cette offre varie avec le temps et - en particulier - peut diminuer et même disparaître, le maintien de la compétitivité implique : 1. le remplacement de l'utilisation des énergies non renouvelables par des énergies renouvelables 2 . le recyclage des produits usés et mis au rebut en vue de la récupération et de la régénération au moins partielle des matières premières utilisées pour son élaboration. Ceci implique le remplacement de l'organisation actuelle de la consommation par une production finale massive de déchets, par une organisation en circuit fermé autant que possible, basée essentiellement sur le recyclage. Ce changement changera profondément la notion de compétitivité et accentuera ses aspects techniques. de la nature et des dimensions des sociétés humaines.

Les deux derniers siècles ont vu le remplacement progressif de nombreuses sociétés humaines locales ou régionales (villes, principautés, états, nations) par des agrégats et des fédérations en groupes beaucoup plus grands - en diversité - en extension - en population. Chacune des sociétés précédentes avait son propre environnement géographique et écologique bien défini et défini - en fonction de la possibilité et de la rapidité des déplacements - en fonction des spécificités de son environnement (voir note sur les civilisations du blé, du riz, etc. L'action humaine a transformé l'esprit radical de ces conditions, et de nouvelles les adaptations sont déjà essentielles. La mutation nécessaire de la notion d'efficacité économique.

La notion actuelle d'efficacité économique a un caractère «endogène». Cela signifie que seuls des critères économiques sont utilisés pour définir la notion d'efficacité. Une telle idée repose implicitement sur la conviction que la science économique peut être établie en faisant abstraction d'au moins certains facteurs non économiques. Ainsi, ce ne sont que partiellement les motivations humaines qui sont prises en compte et que, lorsqu'elles sont prises en compte, seuls les «intérêts» individuels ou collectifs sont considérés en termes de «gains et / ou pertes». En outre, le concept d '«avantage concurrentiel» domine généralement la pensée économique, sauf par exemple l'idée coopérative (où «l'avantage concurrentiel» correspond à l'idée coopérative (où «l'avantage compétitif» correspond au groupe sur les individus ... mais à l'avantage de ceux-ci). Cette façon de voir reflète la situation historique de l'humanité. Jusqu'à nos jours, l'espèce humaine pouvait progresser sans limites vers la plus grande utilisation de son environnement planétaire. De plus, leurs déchets n'avaient pas une signification écologique négative très significative.

Cette situation globale a profondément changé au cours des deux derniers siècles. Au cours de la même période, l'espèce humaine est devenue un facteur dominant dans l'écologie planétaire et a commencé à avoir un impact sérieux sur ses propres conditions globales de survie, en termes écologiques et biologiques. Par conséquent, la notion d'efficacité économique devrait inclure à l'avenir le coût du maintien de l'habitabilité planétaire pour l'espèce humaine. Cela implique la nécessité de maintenir cette même habitabilité pour toutes les espèces vivantes et en particulier pour celles dont 
dépend notre survie. En pratique, cela signifie introduire la notion d'efficacité à un niveau écologique global, beaucoup plus complet, rigoureux et significatif que la simple efficacité économique.

\section{La mécanisation humaine par la technique}

Le processus dominant dans l'évolution humaine au cours des deux derniers siècles a été l'avancée écrasante de toutes les techniques, qui continue à pleine vitesse. Une conséquence peu remarquée est que l'homme (biologique, psychosocial) est devenu une "pièce» d'une immense machine, à l'échelle planétaire, ses activités étant de plus en plus conditionnées par cette machine créée par lui-même. Il semble être une version "géante" et raffinée du termite et des fourmilières du règne animal. Mais un nouvel élément apparaît dont les effets futurs sont encore flous: l'intelligence de chacun des individus participants. D'ailleurs, cette intelligence est aussi généralement conditionnée par le "termitera" humain. La question se pose surtout chez les individus qui parviennent à échapper plus ou moins au conditionnement psychosociologique commun (en général, en changeant de «termitera»). Cependant, dans un nouveau tour de vis, ces "dé-conditionnés" tendent à devenir général à tour de rôle, bien sûr dans le cadre (évolutif) du "termitera".

Ce mécanisme crée un système complexe de self-control socioculturel collectif, qui a fini par individualiser plusieurs cultures dans un monde qui était, loin de l'unification.

\section{LES "DOUZE GRANDS PROBLÈMES"}

\section{Les "douze grands problèmes" (liste provisoire)}

1. Dégradation mondiale des terres arables

2. Avance mondiale des zones désertiques

3. Réchauffement climatique global des climats

4. Pollution planétaire: terrestre, océanique et atmosphérique

5. Utilisation accélérée et épuisement des ressources matérielles non renouvelables

6. Utilisation accélérée et épuisement des ressources énergétiques non renouvelables: charbon, pétrole, gaz

7. Destruction d'espèces végétales et animales multiples, c'est-à-dire patrimoine génétique irremplaçable, et généralement encore inconnu

8. Mutations génétiques de maladies végétales, animales et humaines, avec des risques d'épidémies et de pandémies nouvelles et incontrôlables à court terme.

9. La menace croissante de telles pandémies mondiales se propage par des réseaux de communications mondiales rapides.

10. Accélération de la croissance démographique démographique et technoéconomique qui pourrait surpasser la "capacité de charge humaine planétaire".

11. Amélioration et multiplication des armes de destruction massive, violence et la criminalité au détriment d'une répartition équitable des ressources économiques dans le monde entier.

12. Conflits raciaux, politiques, idéologiques et / ou religieux entraînant guerres et révolutions de plus en plus étendues et destructrices. 


\section{Les "douze grands problèmes" et l'explosion démographique.}

Presque tous les problèmes mentionnés sont des aspects et / ou des résultats directs ou indirects de l'explosion démographique (problème numéro 10) dont les origines doivent être recherchées dans la conjonction des nouvelles technologies, avec la mobilisation massive des énergies fossiles. Ces conditions ont conduit au pillage des ressources de la planète et à la mise en place d'une «idéologie de croissance» (ou «développement») qui semble insoutenable, alors qu'il ne reste presque rien pour piller le «trésor géologique» mis à la portée humaine par nature. Dans un tel moment, la survie des grandes masses humaines apparues au cours des 200 dernières années dépendra des possibilités de transformer la croissance exponentielle de l'économie et la multiplication des hommes dans un régime de stabilité dynamique. Au-delà du pillage aveugle des ressources non renouvelables, ce qui est implicitement remis en question, c'est la réelle «capacité de charge» permanente de la planète en population humaine. Il est hautement probable que le niveau démographique planétaire actuel ne soit pas viable, au rythme actuel de consommation des ressources... Et que nous approchons d'une transition vers un régime de stabilité dynamique fluctuant autour d'un futur niveau soutenable, avec le recyclage des intrants et des sources renouvelables d'énergie. Le problème de la transition est que, si nous dépassons massivement ce niveau, une violente oscillation démographique de relaxation est inévitable.

Une telle transition sera sujette à l'intelligence avec laquelle, au niveau mondial, l'oscillation de la détente est gérée par les dirigeants politiques évitant les famines, les pandémies, le maintien des armes et des mouvements révolutionnaires, la criminalité et les pathologies sociales sous toutes leurs formes.

\section{Les "douze grands problèmes" et le manque de perception des limites.}

Chacun des «12 problèmes» est aussi un aspect ou une conséquence du manque de perception des limites. Ce problème général est d'une grande importance: il résulte de la croyance ou de l'idéologie de la croissance illimitée apparue en Europe au XIXe siècle et répandue dans le monde entier, en particulier dans la seconde moitié du XXe siècle,

Il est clair que toute croissance dépend de sa capacité à capter les ressources de son environnement. Il est également très fréquent que la croissance tende à se poursuivre et à s'accélérer jusqu'à ce qu'elle commence à épuiser son environnement. Au début, il est en général modeste, mais il devient fréquemment exponentiel ou logarithmique parce que, ayant des ressources apparemment inépuisables, il se rétro- alimente de manière accélérée. Cependant, à un certain moment, il y a un tournant, quand le système en croissance commence à produire une baisse significative des ressources disponibles. Il est également remarquable, bien qu'en général il ne soit pas envisagé ni compris que la capacité de recevoir et d'assimiler l'environnement pour des produits utiles - et ceux qui ne le sont pas - est également une ressource cruciale et limitée.

\section{CONCLUSION}

Tout ceci explique pourquoi les courbes de croissance des phénomènes réels montrent des caractéristiques très générales: 1) Croissance au début lente, mais devenant exponentielle vers une limite à l'infini (évidemment inatteignable) et chute soudaine et assez souvent totale, par brusque rupture de tendance à un moment donné et à un moment critique. 2) Croissance logistique, avec l'apparition d'un terme de freinage qui produit une courbe caractérisée par l'approche de plus en plus proche d'un maximum qui ne peut être atteint du tout.

Le point crucial est de comprendre la différence entre ces deux types de croissance et pour comprendre que, tandis que le premier conduit à l'annihilation, seul le second peut conduire à un optimum, généralement plus ou moins fluctuant, par rapport aux conditions environnementales. Métaphoriquement, on pourrait parler de «respiration» du système. Pour la pratique de nos systèmes socio-économiques, cela signifie que - pour éviter un effondrement catastrophique à un moment futur nous devons rechercher et trouver un régime fonctionnel durable à long terme ou à très long terme - 
avec des oscillations de relaxation qui lui donner un caractère de stabilité dynamique fluctuante entre les limites.

Un tel régime dépendra inévitablement des conditions environnementales, et la dynamique de la même chose au fil du temps... L'ignorance éventuelle, ou une mauvaise interprétation de ces conditions, implique une menace invisible, mais elle est catastrophique pour le système. Tout cela constitue à la fois un grand retard et un impératif d'assumer la nécessaire prise de conscience qui conduit à freiner ces pulsions négatives, au profit d'accords permanents, privilégiant une vision globale et des critères écologiques, et la coexistence humaine sur la planète.

\section{REFERENCES}

Collective International (2004). The International Encyclopedia of Systems and Cybernetics, Editor Charles Francois (ALAS, IASCYS, IFSR), K.G. Saur, München, Germany, 2nd edition, 2 vol., 741 p.

\section{BIBLIOGRAPHY}

Charles François is the Honorary President of the Argentine Group for the Study of Systems and Cybernetics (GESI), the Latin American Society of Systemics (ALAS), as well as the Instituto Tecnológico de Buenos Aires (ITBA). He is also a visiting professor in various Universities in Argentina and at the Instituto Andino de Sistemas of Lima in Peru. François is a member of editorial board for the journals Systems Research and Behavioral Science, Systems Practice, and Sistémica. François has worked on researching a sociological understanding of the most significant events of the 20th and 21st centuries, and with the practical management of natural \& man-made complex systems, in systemic and cybernetic terms. François has authored several books, virtual tutorials, bibliographic compilations, and papers in international journals. He is the editor for the International Encyclopedia of Systems and Cybernetics, an encyclopedia for systems theory, cybernetics, complex systems science. He was awarded the Bronze Medal of the American Society for Cybernetics in 2007. He was appointed as the Economic and Commercial Attaché of the Belgian Embassy in Argentina from 1966 to 1987. 